\title{
Analyzing the impact of Social Media Communication Mix on improving Brand Image and enhancing Customers' Experience.
}

\author{
Dr. Kiran. S. Nair.
}

Assistant Professor of Management, Abu Dhabi School of Management, UAE

\begin{abstract}
As brand building is a long-term process the same applies to the universe of social media too. It is said that building a brand image can takes years, but it take a moment to get ruined. Therefore it is very important for organizations to carefully examine all key aspects which is pertinent in building brand image on social media platforms. The real customers' experience for brands on social media significantly influence brand building. The aim of this study is to investigate the effects of social media communication mix on improving brand image and enhancing customers' experience. We identified key factors which help to predict customer behavior and experience with brands in social media platforms and with the same we analyzed the relationship between perceived brand images through customer experience. The sample of this study consists of 225 respondents of mix demographic parameters. To investigate these relationships correlation analysis is used. Strong relevance of relationships were confirmed in two cases with medium dependence out of the seven relationships investigated in this study. Results proves the importance of using social media communication mix for brand building, as these tools got significant ability to influence customers attitude and perception. Social media is the fastest, economical and personal way to communicate efficiently and effectively with the customer. As it happens in real time it can trigger user action instantly if organization has the ability to convey the real emotion. The findings from this study can help the organizations to choose the right social media communication mix for a better and higher return on their marketing investment. This research offers a new perspective in creating social media strategy for building a strong brand image.
\end{abstract}

Keywords: Brand Image, Social media, customer experience, brand management, Communication Mix 